\title{
Association of TLR8 and TLR9 polymorphisms with tuberculosis in a Chinese Han population: a case-control study
}

Ming-Gui Wang, Miao-Miao Zhang, Yu Wang, Shou-Quan Wu, Meng Zhang and Jian-Qing He*

\begin{abstract}
Background: Toll-like receptor (TLR) single nucleotide polymorphisms (SNPS) have been associated with regulation of TLR expression and development of active tuberculosis (TB). The objectives of this study were to determine whether TLR8 and TLR9 SNPs were associated with the development of latent TB infection (LTBI) and the subsequent pulmonary TB (PTB) in a Chinese Han population.

Methods: Two independent samples were enrolled. The first sample contained 584 TB cases and 608 controls; the second sample included 204 healthy controls, 201 LTBI subjects and 209 bacteria-confirmed active PTB patients. Three SNPs (rs3764880, rs187084 and rs5743836) were genotyped. The associations between the SNPs and risk of LTBI or PTB were investigated using unconditional logistic regression analysis.

Results: The A-allele of TLR8 rs3764880 SNP was protective against the development of TB in males (A vs G, OR=0.58, $95 \% \mathrm{Cl}=0.37-0.91)$. The AA genotype of rs3764880 SNP was found to increase the risk of PTB among females with an OR of 4.81 (1.11-20.85). The $G$ allele of TLR9 SNP rs 187084 was found to increase the risk of PTB (G vs A, $P=0.01$, $\mathrm{OR}=1.48,95 \% \mathrm{Cl}=1.10-2.00)$, the significance was also observed under dominant genetic models. The GA-genotype of TLR9 rs 187084 SNP was found to increase the risk of PTB with an OR of 1.68 (1.07-2.65), but was found to decrease the risk of MTB infection with an $\mathrm{OR}=0.64(0.41-0.98)$. TLR9_rs5743836 SNP was excluded from the data analyses, because the minimum allele frequency was $<1 \%$.
\end{abstract}

Conclusions: Our findings in two independent samples indicated that SNPS in TLR8 and TLR9 were associated with the development of TB, and highlight that SNPs may have different effects on disease pathogenesis and progression.

Keywords: Toll-like receptors, Single nucleotide polymorphism, Tuberculosis

\section{Background}

Tuberculosis (TB), caused by infection with Mycobacterium tuberculosis (MTB), remains one of the world's deadliest communicable diseases. According to the World Health Organization, an estimated 10.4 million people developed TB and 1.7 million died of the disease in 2016 [1]. However, most individuals exposed to MTB experience latent MTB infection (LTBI) and do not develop active disease. Considerable evidence suggests

\footnotetext{
* Correspondence: Jianqhe@gmail.com; jianqing_he@scu.edu.cn Department of Respiratory and Critical Care Medicine, West China Hospital, Sichuan University, No. 37, Guo Xue Alley, Chengdu 610041, Sichuan Province, People's Republic of China
}

that host genetic factors play a key role in determining an individual's susceptibility to TB [2-5].

Toll-like receptors (TLRS) are a class of pattern recognition molecules, which are known to play important roles in the innate and adaptive immune system [6], by recognizing pathogen-associated molecular patterns. Most TLRs are expressed on the cell surface, whereas other $\operatorname{TLRs}(3,7,8$, and 9) are expressed intracellularly [7, 8]. TLR7, TLR8, and TLR9 have been implicated in immune diseases due to their ability to recognize oligonucleotide-based (RNA-and DNA-based) molecular patterns as agonists [9].

(c) The Author(s). 2018 Open Access This article is distributed under the terms of the Creative Commons Attribution 4.0 International License (http://creativecommons.org/licenses/by/4.0/), which permits unrestricted use, distribution, and reproduction in any medium, provided you give appropriate credit to the original author(s) and the source, provide a link to the Creative Commons license, and indicate if changes were made. The Creative Commons Public Domain Dedication waiver (http://creativecommons.org/publicdomain/zero/1.0/) applies to the data made available in this article, unless otherwise stated. 
TLR8 is located in the membranes of the endosomal compartment and recognize single-stranded RNA, regulating in the induction of interferon (IFN) and inflammatory cytokines [10-12]. Previous studies have shown that TLR8 variants influence the expression of TLR8 [13-15]. The TLR8 single nucleotide polymorphism (SNP) rs3764880 (Met1 Val) regulates the translation of the two main TLR8 isoforms, and plays a significant part in the immune response $[12,15]$. A study conducted by Davila et al. [14], was the first to demonstrate that SNPs in TLR8 were associated with TB in adults. Since TLR8 is located on chromosome X (Xp22.3-p22.2), males carrying a single copy of the defective allele may have higher risk of TB. In addition, several studies have shown that the $\mathrm{G}$ allele of TLR8 rs3764880 was associated with TB susceptibility in males $[14,16-18]$. These studies demonstrated that the rs3764880 SNP in TLR8 play important roles in TB.

The ligands for TLR9 are DNA-containing CpG motifs [19]. TLR9 is located in the endosomal compartment of plasmacytoid dendritic cells and monocytes/macrophages [19], and plays a vital role in autoimmune diseases and inflammatory diseases by the regulation of type I IFN and inflammatory cytokines [19-21]. The rs187084 and rs5743836 SNPs located in the promoter are the most important and have been associated with various inflammatory diseases [9, 21-24]. Previous functional analyses have shown that both rs187084 and rs5743836 SNPs influence the transcription of TLR9 by regulation of promoter activity $[22,25,26]$. Previous studies indicated that TLR9 is one of the most important receptors in the control of infections with pathogens such as hepatitis C virus [22], Brucella [27], and MTB $[23,28,29]$. Some studies found that the rs187084 in TLR9 showed no association with TB in Vietnam and Iran $[28,30]$. However, no study has explored the association between rs187084 and MTB infection or the process from LTBI to TB. The rs5743836 in TLR9 showed a strong association with tuberculosis in African-Americans and Caucasians [31], while the association was not found in Vietnam [28] or Mexico population [29]. Wu L et al. [32] also reported that rs5743836 was a risk factor for LTBI.

These findings demonstrated that TLR 8 and TLR9 play important roles in infectious diseases, and also emphasized the role of the rs3764880 SNP in TLR8 and rs187084 and rs5743836 SNPs in TLR9. To date, the SNPs of TLR8 and TLR9 have been studied in association with susceptibility to TB, but such studies addressing host genetic susceptibility to TB was limited, whether an association implies susceptibility for developing active disease or just acquisition of MTB infection is unclear. In this study, we investigated the associations of SNPs of TLR8 (rs3764880) and TLR9 (rs187084, rs5743836) with TB in a Chinese Han sample. Then we explored the associations of these SNPs with LTBI or PTB in a second sample of Chinese Han individuals.

\section{Materials and methods Subjects \\ First sample}

A cohort of 584 new TB patients above 15 years of age were recruited from the West China Hospital of Sichuan University. Diagnosis was based on the following criteria: culture positive and/or smear positive for MTB and/or histopathological findings of $\mathrm{TB}$ and clinical and/or radiographic presentation consistent with $\mathrm{TB}$, with positive response to anti-TB therapy. 608 unrelated healthy controls (with unknown status of MTB infection), matched with cases by gender and age, were selected from individuals attending the outpatient department of the West China Hospital for annual physical examination. Healthy controls with a history of prior anti-TB treatment were excluded. All participants diagnosed with diabetes mellitus, human immunodeficiency virus (HIV) co-infection, or in receipt of immunosuppressive therapy, were excluded.

\section{Second sample}

A cohort of 614 participants were recruited: patients with pulmonary TB (PTB) $(n=209)$, LTBI subjects $(n=201)$, and healthy controls $(\mathrm{HC})$ without MTB infection $(n=204)$. As with the first sample, the subjects in the second sample were recruited from the West China Hospital in Chengdu. Eligibility criteria for PTB patients included: 1) $\geq 18$ years old; 2) diagnosis of PTB based on sputum smear examinations for acid-fast bacilli and/or the culture of MTB. Both LTBI and $\mathrm{HC}$ subjects were unrelated and asymptomatic contacts of bacteria-confirmed active TB patients. LTBI was defined as a positive result for the QuantiFERON-TB Gold In-Tube and $\mathrm{HC}$ was defined as a negative result for the same assay, and patients belonging to both subgroups had no radiological evidence of active TB, negative sputum smear and culture, and no history of TB. Exclusion criteria included a positive serological test for HIV infection, organ transplantation, primary immunodeficiency, cancer, and treatment with immunosuppressive drugs, endocrine disorders such as diabetes, autoimmune or chronic renal disease, and extrapulmonary TB cases.

All protocols were approved by the ethics committee of the West China Hospital of Sichuan University. Written informed consent was obtained from all participants involved in this study. Demographic characteristics of all participants were collected from a detailed questionnaire.

\section{Genotyping}

A volume of 2-5 ml blood samples were collected in EDTA tubes (BD Vacutainers, Franklin Lakes, NJ, USA) from each participant. Genomic DNA was extracted from 
whole blood following a protocol described elsewhere [33]. Three SNPs (rs3764880, rs187084, rs5743836) were genotyped using a custom-by-design $2 \times 48$-Plex SNPscan $^{\text {Ts }}$ Kit (Cat\#: G0104, Genesky Biotechnologies Inc., Shanghai, China) as described previously [34]. This kit was developed according to a patented SNP genotyping technology by Genesky Biotechnologies Inc., which was based on double ligation and multiplex fluorescence PCR. As a quality control measure, $5 \%$ of the samples were genotyped in duplicate using the same method to check for concordance. Assessment of genotypes was done by laboratory personnel without any prior knowledge of the diagnosis of the subjects.

\section{Statistical analyses}

The clinical and demographic characteristics were compared using the student's test or ANOVA for continuous variables and with the $\chi^{2}$ test or Fisher's exact test for categorical variables. $P<0.05$ was considered significant.

Hardy-Weinberg Equilibrium (HWE) in healthy controls was tested using asymptotic Pearson's chi-square tests for each SNP, in each study. Because the TLR8 rs3764880 SNP is located on chromosome $\mathrm{X}$, we tested for HWE in females only. Since we used loose-matching to select cases and controls, associations between polymorphisms and risk of TB, LTBI or PTB were investigated using unconditional logistic regression analysis [35], adjusting for age and gender, and smoking as in the analysis of the first sample. Because this was an exploratory analysis, we did not introduce a correction for multiple comparisons. The results were expressed by odds ratios (ORs) and 95\% confidence intervals (95\% CIs). All statistical analyses were carried out using SPSS statistical software, release 19.0.

\section{Results}

\section{Clinical information of study subjects}

Demographic and clinical parameters of the patients are summarized in Table 1. As shown in Table 1, $584 \mathrm{~TB}$ cases and 608 controls from the Chinese Han population were enrolled in the first sample, with no significant difference in age and gender ratio. More smokers were found among the TB patients as compared with controls $(P=0.012) .614$ eligible participants were enrolled in the second sample, including 209 PTB cases, 201 LTBI, and 204 HC (Table 1). There was no significant difference in gender among the three groups, while the mean age was significantly different among the PTB, LTBI and control groups $(P<0.001)$. No deviations from HWE were detected in all groups $(P>0.05)$. Genotyping data for the three SNPs were successfully obtained for $\geq 98.8 \%$ of the subjects. However, TLR9_rs5743836 SNP was excluded from the data analyses, because the minimum allele frequency (MAF) was $\leq 1 \%$.

\section{TLR8 rs3764880 and TB disease progression}

The genotype and allele distributions of this SNP in the first sample are shown in Table 2. Firstly, a decreased frequency of the minor allele A of TLR8_rs3764880 was found in the male TB patient group, and found to be protected against TB $(\mathrm{OR}=0.58,95 \% \mathrm{CI}=0.37-0.91, P=0.02)$ after adjusting for age and smoking in the first sample. No difference was found in the genotype frequencies among male patients in this sample. There was no difference in both allele and genotype frequencies among female subjects in the first sample. Further analysis was performed in the second sample (Table 3). No significant differences in the allele frequency of TLR8_rs3764880 SNP were observed for male or female subjects when comparing PTB disease with LTBI groups or when comparing LTBI group with $\mathrm{HC}$ subjects. In contrast, an increased frequency of the AA genotype of rs3764880 was observed in female patients, and found to increase the risk of PTB $(\mathrm{OR}=4.81$, $95 \% \mathrm{CI}=1.11-20.85, P=0.04)$ when comparing PTB disease with LTBI groups. No differences in frequencies of TLR8_rs3764880 genotypes were observed for male or female patients when comparing LTBI group with HC subjects.

\section{TLR9 rs 187084 and TB disease progression}

The genotype and allele distributions of this SNP in the first and second samples are shown in Table 4. Associations between TLR9 rs187084 and the development of TB, PTB or LTBI were assessed in dominant, and recessive models. Firstly, analysis in the first sample detected no difference in allele and genotype frequencies among TB patients and controls. No significant differences were

Table 1 Clinical characteristics of the study population

\begin{tabular}{|c|c|c|c|c|c|c|c|}
\hline & \multicolumn{3}{|l|}{ First sample } & \multicolumn{4}{|c|}{ Second sample } \\
\hline & TB patients (584) & Control (608) & $P$ value & PTB (209) & LTBI (201) & HC (204) & $P$ value \\
\hline Age $($ mean $\pm S D)$ & $36.68 \pm 15.61$ & $37.18 \pm 15.68$ & 0.608 & $38.76 \pm 16.97$ & $49.09 \pm 15.91$ & $45.71 \pm 14.90$ & $<0.001$ \\
\hline \multicolumn{8}{|l|}{ Gender } \\
\hline Males, n & 299 & 302 & 0.598 & 107 & 95 & 93 & 0.503 \\
\hline Females, $\mathrm{n}$ & 285 & 306 & & 102 & 106 & 111 & \\
\hline Smokers, n(\%) & 173 (29.62\%) & 141 (23.19\%) & 0.012 & - & - & - & - \\
\hline
\end{tabular}

Abbreviations: TB tuberculosis, PTB pulmonary tuberculosis, LTBI latent tuberculosis infection, $H C$ healthy control 
Table 2 The genotype and allele frequencies of rs3764880 in patients with TB and controls

\begin{tabular}{|c|c|c|c|c|}
\hline Genotypes and allele frequencies & Cases, n (\%) & Controls, n (\%) & OR $(95 \% \mathrm{Cl})$ & $P$ value \\
\hline \multicolumn{5}{|l|}{ Males } \\
\hline TLR8 rs3764880 major allele G & $253(86.6)$ & $236(79.5)$ & 1 & Reference \\
\hline TLR8 rs3764880 minor allele A & $39(13.4)$ & $61(20.5)$ & $0.58(0.37-0.91)$ & 0.02 \\
\hline \multicolumn{5}{|l|}{ Females } \\
\hline TLR8 rs3764880 GG & $203(71.2)$ & $209(68.8)$ & 1 & Reference \\
\hline TLR8 rs3764880 GA & $76(26.7)$ & $82(27.0)$ & $0.93(0.65-1.35)$ & 0.71 \\
\hline TLR8 rs3764880 AA & $6(2.1)$ & $13(4.3)$ & $0.49(0.18-1.31)$ & 0.16 \\
\hline TLR8 rs3764880 major allele G & $482(84.6)$ & $500(82.2)$ & 1 & Reference \\
\hline TLR8 rs3764880 minor allele A & $88(15.4)$ & $108(17.8)$ & $0.84(0.61-1.14)$ & 0.26 \\
\hline
\end{tabular}

Abbreviations: $T B$ tuberculosis, $O R$ odds ratio, $C l$ confidence interval. $\mathrm{P}$ value adjusted for age and smoking

detected in TLR9 rs187084 SNP between TB patients and controls under any genetic model in the first sample. To further explore whether this SNP was associated with susceptibility to PTB or MTB infection, the second sample was analyzed. When comparing PTB patients with LTBI group, significant associations with developing disease were observed after adjusting for age and gender (Table 4). The GA-genotype of TLR9 rs187084 SNP was found more frequent in PTB cases, and was found to increase the risk of PTB with an OR of 1.68 (95\% CI: 1.07-2.65). The prevalence of minor allele G of rs187084 was significantly higher in patients with
PTB than that of controls $(41.9 \%$ vs $32.3 \%$, OR $=1.48$, 95\% $\mathrm{CI}=1.10-2.00, \quad P=0.01)$. The significance was also observed under dominant genetic models (Table 4), indicating that the GG and GA genotypes increased risk susceptibility to develop active PTB from LTBI status. When comparing the LTBI group with HC subjects, a significant difference was found in genotype distributions. GA-genotype was found to decrease the risk of MTB infection (GA vs AA, $P=0.04, \mathrm{OR}=0.64,95 \% \mathrm{CI}=0.41$ 0.98). However, no differences in allele distributions or genotype distributions under different genetic models were observed between LTBI group and HC subjects.

Table 3 The genotype and allele frequencies of rs3764880 in the second sample

\begin{tabular}{|c|c|c|c|c|}
\hline Genotypes and allele frequencies & Cases, n (\%) & Controls, n (\%) & OR $(95 \% \mathrm{Cl})$ & $P$ value \\
\hline \multicolumn{5}{|l|}{ PTB and LTBI } \\
\hline \multicolumn{5}{|l|}{ Males } \\
\hline TLR8 rs3764880 major allele $\mathrm{G}$ & $84(79.2)$ & $84(88.4)$ & 1 & Reference \\
\hline TLR8 rs3764880 minor allele A & $22(20.8)$ & $11(11.6)$ & $1.92(0.85-4.32)$ & 0.12 \\
\hline \multicolumn{5}{|l|}{ Females } \\
\hline TLR8 rs3764880 GG & $65(63.7)$ & $71(67.6)$ & 1 & Reference \\
\hline TLR8 rs3764880 GA & $28(27.5)$ & $31(29.5)$ & $1.08(0.56-2.09)$ & 0.83 \\
\hline TLR8 rs3764880 AA & $9(8.8)$ & $3(2.9)$ & $4.81(1.11-20.85)$ & 0.04 \\
\hline TLR8 rs3764880 major allele $\mathrm{G}$ & $158(77.5)$ & $173(82.4)$ & 1 & Reference \\
\hline TLR8 rs3764880 minor allele A & $46(22.5)$ & 37 (17.6) & $1.56(0.93-2.62)$ & 0.09 \\
\hline \multicolumn{5}{|l|}{ LTBI and HC } \\
\hline \multicolumn{5}{|l|}{ Males } \\
\hline TLR8 rs3764880 major allele $\mathrm{G}$ & $84(88.4)$ & $76(82.6)$ & 1 & Reference \\
\hline TLR8 rs3764880 minor allele A & $11(11.6)$ & $16(17.4)$ & $0.53(0.23-1.25)$ & 0.15 \\
\hline \multicolumn{5}{|l|}{ Females } \\
\hline TLR8 rs3764880 GG & $71(67.6)$ & $70(63.1)$ & 1 & Reference \\
\hline TLR8 rs3764880 GA & $31(29.5)$ & $36(32.4)$ & $0.85(0.47-1.52)$ & 0.58 \\
\hline TLR8 rs3764880 AA & $3(2.9)$ & $5(4.5)$ & $0.64(0.15-2.84)$ & 0.56 \\
\hline TLR8 rs3764880 major allele $\mathrm{G}$ & $173(82.4)$ & $176(79.3)$ & 1 & Reference \\
\hline TLR8 rs3764880 minor allele A & $37(17.6)$ & $46(20.7)$ & $0.83(0.51-1.34)$ & 0.44 \\
\hline
\end{tabular}




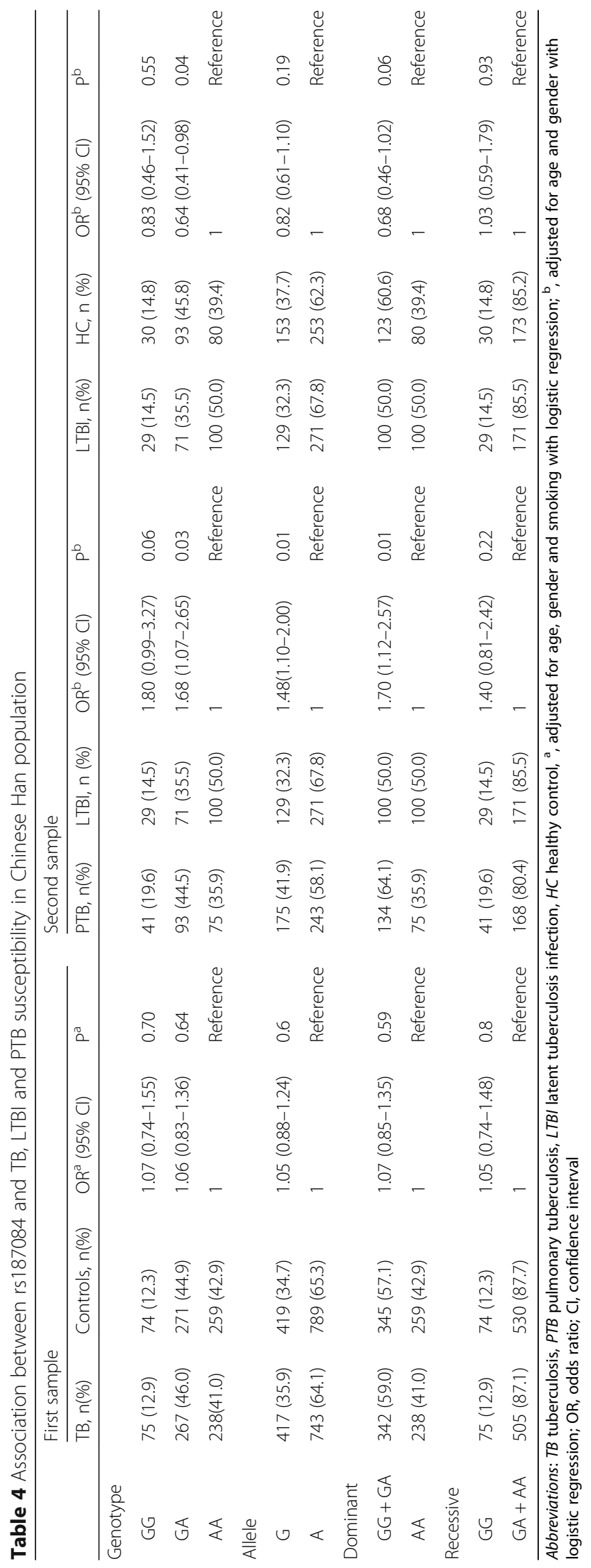




\section{Discussion}

Multiple groups have investigated associations between the three SNPs which we examined in the in current study and TB susceptibility in a variety of populations, but the results were inconsistent $[14,17,18,28,30,32$, 36-40]. Few studies have classified LTBI and HC without MTB infection. The current study first analyzed the association between TLR8/9 SNPs and TB in the first sample, and then further explored the TLR8/9 variants with LTBI and active PTB in the second sample. Our results suggest that genetic variants might have different roles in the development of active PTB and LTBI.

Davila et al. [14] for the first time found that four SNPs (rs3764879G/C, rs3788935G/A, rs3761624G/A, rs3764880G/A) in TLR8 showed evidence of association with TB susceptibility with minor alleles showing an increased susceptibility to PTB in males in Russian and Indonesian populations. Furthermore, associations have also been found in Turkish male children [17], Pakistan population [41], and South African population [18]. However, neither Kobayashi et al. [42] nor Chimusa et al. [43] showed any association between rs3764880 and TB susceptibility. Our results showed that the rs3764880 A allele in TLR8 greatly reduced TB risk in males. This result was consistent with that in a Pakistan population [41], which showed the rs3764880 A allele had a protective role against TB,but different from that in Turkish [17], South African [18], Russian and Indonesian populations [14], which showed the rs3764880 A allele increased susceptibility to TB in males. Subsequently, our data in the second sample provided new evidence for our understanding of the role of rs3764880 in the development of LTBI and PTB. The data suggested that the AA-genotype of rs3764880 increased susceptibility to PTB among females, however, might decrease susceptibility to LTBI. The difference between our results and that of other studies may be due to differences in race, or living environment. Therefore, the detection of this SNP among LTBI subjects may provide important information in the assessment of their risk profiles for susceptibility to development of PTB. Previous studies have found that the SNPs in TLR8 might have gender effects across the genetic association studies on TB susceptibility $[14,17,18,41]$. Our results also found a gender difference: male carriers of rs3764880 allele A showed a decreased risk for $\mathrm{TB}$, and females carrying the AA genotype increased the risk of PTB when compared with LTBI. What's more, this gender effect was both demonstrated in the two independent study samples.

Several investigators have studied the roles of TLR9 SNPs in TB. As reported previously in studies of Vietnam and Iran [28, 30], we found the rs187084 in TLR9 showed no association with TB in the first sample. In the second sample, the G-allele of TLR9 rs187084 greatly increased
PTB risk, and this association was also observed under a dominant genetic model, which suggested a risk role of the allele G. However, the GA genotype decreased the risk of MTB infection. A study by Digna Rosa Velez et al. [31] showed a strong association between rs5743836 and tuberculosis in African-Americans and Caucasians population, while the association was not found in Vietnam [28] or Mexico population [29]. Another study conducted by Wu L et al. [32] showed that rs5743836 was a risk factor for LTBI and its MAF was 0.27 in a Chinese population in Shanghai city. The MAF of this SNP is high among African-Americans, Africans and Caucasians [31], while low in the Mexican population [29] and Vietnamese people [28]. These studies, thus indicated that the MAF of rs5743836 may vary between different ethnic groups. In this study, we found the MAF of rs5743836 was less than $1 \%$. Since our sample size may not be large enough for a SNP with low MAF, and may potentially lead to false associations with the phenotype investigated, the rs5743836 was excluded in the data analyses. The MAF of rs5743836 in Beijing Han population from the International HapMap Project (http://www.1000genomes.org/) is less than 0.01, which support our result and indicates a low mutation rate in Chinese Han population. Therefore, the higher rs5743836 MAF in the study of Wu L et al. may suggest that populations other than Chinese Han were included in the study [32].

It has been discussed that some SNPs may actually be associated with MTB infection [36, 37], some may be associated with active TB [38, 39] and other studies suggested some SNPs may have different impacts on the susceptibility to LTBI and PTB $[32,40,44]$. Lu et al. found that an immunity-related GTPase family M SNP was associated with active TB and LTBI, but also plays opposite roles in the development of active TB and LTBI [44]. Our results provide strong evidence that the allele "A" of the polymorphism rs3764880 was associated with decreased risk of tuberculosis in the first sample. The genotype "AA" of rs3764880 was more commonly found in female PTB patients compared with LTBI, indicating this genotype increases the possibility of progression of tuberculosis infection to disease in the second sample. For the polymorphism of rs187084 in TLR9, we found the genotype "GA" and allele "G" was more common in PTB patients compared with LTBI, which demonstrated that this allele increased the risk of progression from tuberculosis infection to active disease. However, the GA-genotype was found to reduce the risk of MTB infection, thereby indicating that the results of our two samples were inconsistent and may have been caused by a number of factors. It may be caused by the following reasons. First, we included all TB patients in our first sample without differentiating between the different types of TB, while we only included PTB patients in the 
second sample. Genetic polymorphisms may have different effects on PTB and extra-pulmonary TB (EPTB), which may be due to the fact that different immune mechanisms are involved in PTB and EPTB [45]. Second, the results in the first sample may be explained, when the distinction between LTBI and HC without the infection of MTB is ignored. In other words, the results may be different because of the differences within the control group. Therefore, it is reasonable that these SNPs showed different results in the two samples.

Our study faced some intrinsic limitations. First, small sample size in the second sample may have limited our ability to detect potential influence of TLR SNPs on the susceptibility to both LTBI and PTB. Further studies are therefore necessary to validate these associations in larger sample sizes and other populations. Second, TB case of the first sample included both clinical diagnosed and bacteria confirmed TB patients, regardless of their types, which could potentially reduce the validity of our conclusions. Third, LTBI and HC subjects included in the second sample were asymptomatic contacts of bacteriaconfirmed active TB patients, the assessment of exposure to an active TB case relied on self-reported behavior, which may not be accurate among participants and may cause misclassification bias (once exposed, those controls could become cases). Forth, it is likely that linkage disequilibrium patterns differed among other populations and the Han Chinese population in this study, and thus, those associations may not replicate exactly [14, $28,31]$. A plausible explanation is that the SNPs that we identified are in linkage disequilibrium with another mutation that either confers a functional or a regulatory change within those genes. Hence, to interpret results across studies, future experiments should cover all common variation in the associated TLR8/9 gene region in order to pinpoint the causal polymorphism. Furthermore, one potential limitation in our study was a lack of correction for multiple comparisons. The reported statistically significant results would need to be regarded as nominally significant.

\section{Conclusion}

Our study provides another evidence supporting the importance of host genetic variability in TB susceptibility. We identified the allele A of the rs3764880 that appears protective against $\mathrm{TB}$ among males of Chinese Han ancestry. Our results also highlight that polymorphisms may have different effects on disease pathogenesis and progression. This study may help to identify those TB-affected individuals most susceptible to disease, and to improve our understanding of the effects of genetic heterogeneity on the development of the different stages of $\mathrm{TB}$.

\section{Abbreviations}

Cls: Confidence intervals; EPTB: extra-pulmonary tuberculosis; HC: Healthy controls; HIV: Human immunodeficiency virus; HWE: Hardy-Weinberg Equilibrium; IFN: Interferon; LTBI: Latent Mycobacterium tuberculosis infection; MAF: Minimum allele frequency; MTB: Mycobacterium tuberculosis; ORs: Odds ratios; PTB: Pulmonary Tuberculosis; SNP: Single nucleotide polymorphism; TB: Tuberculosis; TLRs: Toll-like receptors

\section{Acknowledgements}

Not applicable.

\section{Funding}

This work was supported by the Research Fund for the Doctoral Program of Higher Education of China [grant number 20130181110068], the National Natural Science Foundation of China [grant number 81170042 , grant number 81370121], and the National Scientific and Technological Major Project of China [grant number 2012ZX10004-901].

\section{Availability of data and materials}

The datasets used and analyzed during the current study are available from the corresponding author upon reasonable request.

\section{Authors' contributions}

MGW performed statistical analysis, wrote the manuscript, and was involved in the interpretation of findings. MMZ developed study protocol, collected data, and supervised this study. Y W developed study protocol and collected data. SQW was involved in data analysis and manuscript revision. MZ developed study protocol and supervised the study. JQH developed study protocol, collected data, revised the manuscript, and interpreted the findings. All authors read and approved the final manuscript.

\section{Ethics approval and consent to participate}

All protocols were approved by the ethics committee of the West China Hospital of Sichuan University. Written informed consent was obtained from all participants or their legal guardians.

Consent for publication

Not applicable.

\section{Competing interests}

The authors declare that they have no competing interests.

\section{Publisher's Note}

Springer Nature remains neutral with regard to jurisdictional claims in published maps and institutional affiliations.

Received: 10 May 2018 Accepted: 31 October 2018

Published online: 13 November 2018

\section{References}

1. World Health Organization. Global Tuberculosis Report 2017. Geneva: World Health Organization; 2017.

2. Bellamy R. Genetic susceptibility to tuberculosis. Clin Chest Med. 2005;26(2): 233-46 vi

3. Casanova JL, Abel L. Genetic dissection of immunity to mycobacteria: the human model. Annu Rev Immunol. 2002;20:581-620.

4. Baghdadi JE, Orlova M, Alter A, Ranque B, Chentoufi M, Lazrak F, et al. An autosomal dominant major gene confers predisposition to pulmonary tuberculosis in adults. J Exp Med. 2006;203(7):1679-84.

5. Fortin A, Abel L, Casanova JL, Gros P. Host genetics of mycobacterial diseases in mice and men: forward genetic studies of BCG-osis and tuberculosis. Annu Rev Genomics Hum Genet. 2007;8:163-92.

6. Aderem A, Ulevitch RJ. Toll-like receptors in the induction of the innate immune response. Nature. 2000;406(6797):782-7.

7. Pandey S, Agrawal DK. Immunobiology of toll-like receptors: emerging trends. Immunol Cell Biol. 2006;84(4):333-41.

8. Takeda K, Kaisho T, Akira S. Toll-like receptors. Annu Rev Immunol. 2003;21: $335-76$.

9. Krieg AM, Vollmer J. Toll-like receptors 7, 8, and 9: linking innate immunity to autoimmunity. Immunol Rev. 2007;220:251-69. 
10. Hornung $V$, Barchet W, Schlee M, Hartmann G. RNA recognition via TLR7 and TLR8. Handb Exp Pharmacol. 2008;183:71-86.

11. Heil F, Hemmi H, Hochrein H, Ampenberger F, Kirschning C, Akira S, et al. Species-specific recognition of single-stranded RNA via toll-like receptor 7 and 8. Science. 2004;303(5663):1526-9.

12. Oh DY, Taube S, Hamouda O, Kucherer C, Poggensee G, Jessen H, et al. A functional toll-like receptor 8 variant is associated with HIV disease restriction. J Infect Dis. 2008;198(5):701-9.

13. Wang $\mathrm{CH}$, Eng HL, Lin KH, Liu HC, Chang CH, Lin TM. Functional polymorphisms of TLR8 are associated with hepatitis $C$ virus infection. Immunology. 2014;141(4):540-8.

14. Davila S, Hibberd ML, Hari Dass R, Wong HE, Sahiratmadja E, Bonnard C, et al. Genetic association and expression studies indicate a role of toll-like receptor 8 in pulmonary tuberculosis. PLoS Genet. 2008;4(10):e1000218.

15. Gantier MP, Irving AT, Kaparakis-Liaskos M, Xu D, Evans VA, Cameron PU, et al. Genetic modulation of TLR8 response following bacterial phagocytosis. Hum Mutat. 2010;31(9):1069-79.

16. Lai YF, Lin TM, Wang CH, Su PY, Wu JT, Lin MC, et al. Functional polymorphisms of the TLR7 and TLR8 genes contribute to Mycobacterium tuberculosis infection. Tuberculosis (Edinb). 2016;98:125-31.

17. Dalgic N, Tekin D, Kayaalti Z, Cakir E, Soylemezoglu T, Sancar M. Relationship between toll-like receptor 8 gene polymorphisms and pediatric pulmonary tuberculosis. Dis Markers. 2011:31(1):33-8.

18. Salie M, Daya M, Lucas LA, Warren RM, van der Spuy GD, van Helden PD, et al. Association of toll-like receptors with susceptibility to tuberculosis suggests sex-specific effects of TLR8 polymorphisms. Infect Genet Evol. 2015:34:221-9.

19. Hemmi H, Takeuchi $O$, Kawai T, Kaisho T, Sato S, Sanjo H, et al. A toll-like receptor recognizes bacterial DNA. Nature. 2000;408(6813):740-5.

20. Misch EA, Hawn TR. Toll-like receptor polymorphisms and susceptibility to human disease. Clin Sci (Lond). 2008;114(5):347-60.

21. Baccala R, Hoebe K, Kono DH, Beutler B, Theofilopoulos AN. TLR-dependent and TLR-independent pathways of type I interferon induction in systemic autoimmunity. Nat Med. 2007;13(5):543-51.

22. Fischer J, Weber ANR, Bohm S, Dickhofer S, El Maadidi S, Deichsel D, et al. Sex-specific effects of TLR9 promoter variants on spontaneous clearance of HCV infection. Gut. 2017;66(10):1829-37.

23. Bafica A, Scanga CA, Feng CG, Leifer C, Cheever A, Sher A. TLR9 regulates Th1 responses and cooperates with TLR2 in mediating optimal resistance to Mycobacterium tuberculosis. J Exp Med. 2005;202(12):1715-24.

24. Schurz H, Daya M, Moller M, Hoal EG, Salie M. TLR1, 2, 4, 6 and 9 variants associated with tuberculosis susceptibility: a systematic review and metaanalysis. PLoS One. 2015;10(10):e0139711.

25. Ng MT, Van't Hof R, Crockett JC, Hope ME, Berry S, Thomson J, et al. Increase in NF-kappaB binding affinity of the variant $C$ allele of the toll-like receptor 9 -1237T/C polymorphism is associated with helicobacter pyloriinduced gastric disease. Infect Immun. 2010;78(3):1345-52.

26. Hamann L, Glaeser C, Hamprecht A, Gross M, Gomma A, Schumann RR. Toll-like receptor (TLR)-9 promotor polymorphisms and atherosclerosis. Clin Chim Acta. 2006;364(1-2):303-7.

27. Copin R, De Baetselier P, Carlier Y, Letesson JJ, Muraille E. MyD88-dependent activation of B220-CD11b+LY-6C+ dendritic cells during Brucella melitensis infection. J Immunol. 2007:178(8):5182-91.

28. Graustein AD, Horne DJ, Arentz M, Bang ND, Chau TT, Thwaites GE, et al. TLR9 gene region polymorphisms and susceptibility to tuberculosis in Vietnam. Tuberculosis (Edinb). 2015;95(2):190-6.

29. Torres-Garcia D, Cruz-Lagunas A, Garcia-Sancho Figueroa MC, FernandezPlata R, Baez-Saldana R, Mendoza-Milla C, et al. Variants in toll-like receptor 9 gene influence susceptibility to tuberculosis in a Mexican population. J Transl Med. 2013:11:220.

30. Jahantigh D, Salimi S, Alavi-Naini R, Emamdadi A, Owaysee Osquee H, Farajian Mashhadi F. Association between TLR4 and TLR9 gene polymorphisms with development of pulmonary tuberculosis in Zahedan, southeastern Iran. ScientificWorldJournal. 2013;2013:534053.

31. Velez DR, Wejse C, Stryjewski ME, Abbate E, Hulme WF, Myers JL, et al. Variants in toll-like receptors 2 and 9 influence susceptibility to pulmonary tuberculosis in Caucasians, African-Americans, and west Africans. Hum Genet. 2010;127(1):65-73.

32. Wu L, Hu Y, Li D, Jiang $W, X u B$. Screening toll-like receptor markers to predict latent tuberculosis infection and subsequent tuberculosis disease in a Chinese population. BMC Med Genet. 2015;16:19.
33. Liu Q, Wu S, Xue M, Sandford AJ, Wu J, Wang Y, et al. Heterozygote advantage of the rs3794624 polymorphism in CYBA for resistance to tuberculosis in two Chinese populations. Sci Rep. 2016;6:38213.

34. Du W, Cheng J, Ding H, Jiang Z, Guo Y, Yuan H. A rapid method for simultaneous multi-gene mutation screening in children with nonsyndromic hearing loss. Genomics. 2014;104(4):264-70.

35. Kuo CL, Duan Y, Grady J. Unconditional or conditional logistic regression model for age-matched case-control data? Front Public Health. 2018;6:57.

36. Horne DJ, Graustein AD, Shah JA, Peterson G, Savlov M, Steele S, et al. Human ULK1 variation and susceptibility to Mycobacterium tuberculosis infection. J Infect Dis. 2016:214(8):1260-7.

37. Nonghanphithak D, Reechaipichitkul W, Namwat W, Lulitanond V, Naranbhai V, Faksri K. Genetic polymorphisms of CCL2 associated with susceptibility to latent tuberculous infection in Thailand. Int J Tuberc Lung Dis. 2016:20(9):1242-8.

38. Lu Y, Zhu Y, Wang X, Wang F, Peng J, Hou H, et al. FOXO3 rs12212067: $\mathrm{T}>\mathrm{G}$ association with active tuberculosis in Han Chinese population. Inflammation. 2016;39(1):10-5.

39. Dalgic N, Tekin D, Kayaalti Z, Soylemezoglu T, Cakir E, Kilic B, et al. Arg753Gln polymorphism of the human toll-like receptor 2 gene from infection to disease in pediatric tuberculosis. Hum Immunol. 2011;72(5):440-5.

40. Stein CM, Zalwango S, Chiunda AB, Millard C, Leontiev DV, Horvath AL, et al Linkage and association analysis of candidate genes for TB and TNFalpha cytokine expression: evidence for association with IFNGR1, IL-10, and TNF receptor 1 genes. Hum Genet. 2007;121(6):663-73.

41. Bukhari M, Aslam MA, Khan A, Iram Q, Akbar A, Naz AG, et al. TLR8 gene polymorphism and association in bacterial load in southern Punjab of Pakistan: an association study with pulmonary tuberculosis. Int J Immunogenet. 2015;42(1):46-51.

42. Kobayashi K, Yuliwulandari R, Yanai H, Naka I, Lien LT, Hang NT, et al. Association of TLR polymorphisms with development of tuberculosis in Indonesian females. Tissue Antigens. 2012;79(3):190-7.

43. Chimusa ER, Zaitlen N, Daya M, Moller M, van Helden PD, Mulder NJ, et al. Genome-wide association study of ancestry-specific TB risk in the south African Coloured population. Hum Mol Genet. 2014;23(3):796-809.

44. Lu Y, Li Q, Peng J, Zhu Y, Wang F, Wang C, et al. Association of autophagyrelated IRGM polymorphisms with latent versus active tuberculosis infection in a Chinese population. Tuberculosis (Edinb). 2016;97:47-51.

45. Majorov KB, Lyadova IV, Kondratieva TK, Eruslanov EB, Rubakova El, Orlova $\mathrm{MO}$, et al. Different innate ability of I/St and a/Sn mice to combat virulent Mycobacterium tuberculosis: phenotypes expressed in lung and Extrapulmonary macrophages. Infect Immun. 2003;71(2):697-707.

Ready to submit your research? Choose BMC and benefit from:

- fast, convenient online submission

- thorough peer review by experienced researchers in your field

- rapid publication on acceptance

- support for research data, including large and complex data types

- gold Open Access which fosters wider collaboration and increased citations

- maximum visibility for your research: over $100 \mathrm{M}$ website views per year

At $\mathrm{BMC}$, research is always in progress.

Learn more biomedcentral.com/submission 